\title{
Control Strategies of Multiple Current-Source PWM Converters for a 20MW SMES System
}

\author{
Member Kazuhiro Imaie (Hitachi works, Hitachi LTD.) \\ Member Osami Tsukamoto (Yokohama National University) \\ Non-Member Sakae Washida (Electric Power Development Co., Ltd.)
}

\begin{abstract}
Control strategies for multiple current-source converters ${ }^{[1]}$ employing large capacity gate turn-off (GTO) thyristors are applied for a $20 \mathrm{MW}$ Superconducting Magnetic Energy Storage (SMES) system. Suitable control method and control block diagrams are proposed by taking much use of the multiple structure. Pulse Width Modulation (PWM) control method of current source converters for the purpose of high efficiency and low harmonic distortion is explained. A unique control method for small power output is shown. The results of digital simulation by Electro Magnetic Transient Program (EMTP) are provided to validate the proposed control methods of SMES power conditioning.
\end{abstract}

Key words: SMES, PQ control, PWM, Current-Source Converters, and EMTP

\section{Introduction}

Multiple structured converters are used with the increase in capacity of power conditioning system. There are two types of circuits available, AC direct connected multiplication ${ }^{[2]}$ and transformer multiplication. Former one is useful when the number of converters is increased and the later is effective when small number of converters is required. The transformer multiplication would be available for both the capacity growth and the low order harmonics reduction with small number of converters.

SMES system would be a valuable system for storing electric energy with high efficiency. SMES-based power conditioning system by static power converters should be applicable for rapid power conditioning of the power transmission line to increase the stability by supplying short-term peak energy and by compensating generation shortage.

In this paper, it is shown the multiple current source converters for the 20MW SMES pilot plant that utilize $6 \mathrm{kV}-3 \mathrm{kA}$ GTO thyristor, and also examined the control strategies suitable for practical applications. For practical SMES applications, several control methods as well as a four-quadrant of $\mathrm{PQ}$ control are necessary. 5 pulse-PWM control method is presented to meet the requirement of both minimum loss and low distortion for the power system. A small power output control is proposed with the advantage of the multi-structure to cover the range that a conventional single converter cannot control because of the restricted minimum pulse width of GTO for the protection reason. The digital simulations are examined previous to a large-scale pilot plant. They will give the feasibility of the control strategies by showing the EMTP simulation results. Then the proposed converters, the schematic block diagram, the principles of the control methods and simulation results are explained in detail in following chapters.

\section{Main Circuit Configuration}

The configuration of the proposed SMES System is shown in fig. 1. There are four current-source 3-phase converters in the SMES system; each converter includes a 6-arm bridge and a transformer. Each arm of the converter consists of four $6 \mathrm{kV}-3 \mathrm{kA}$ GTOs'

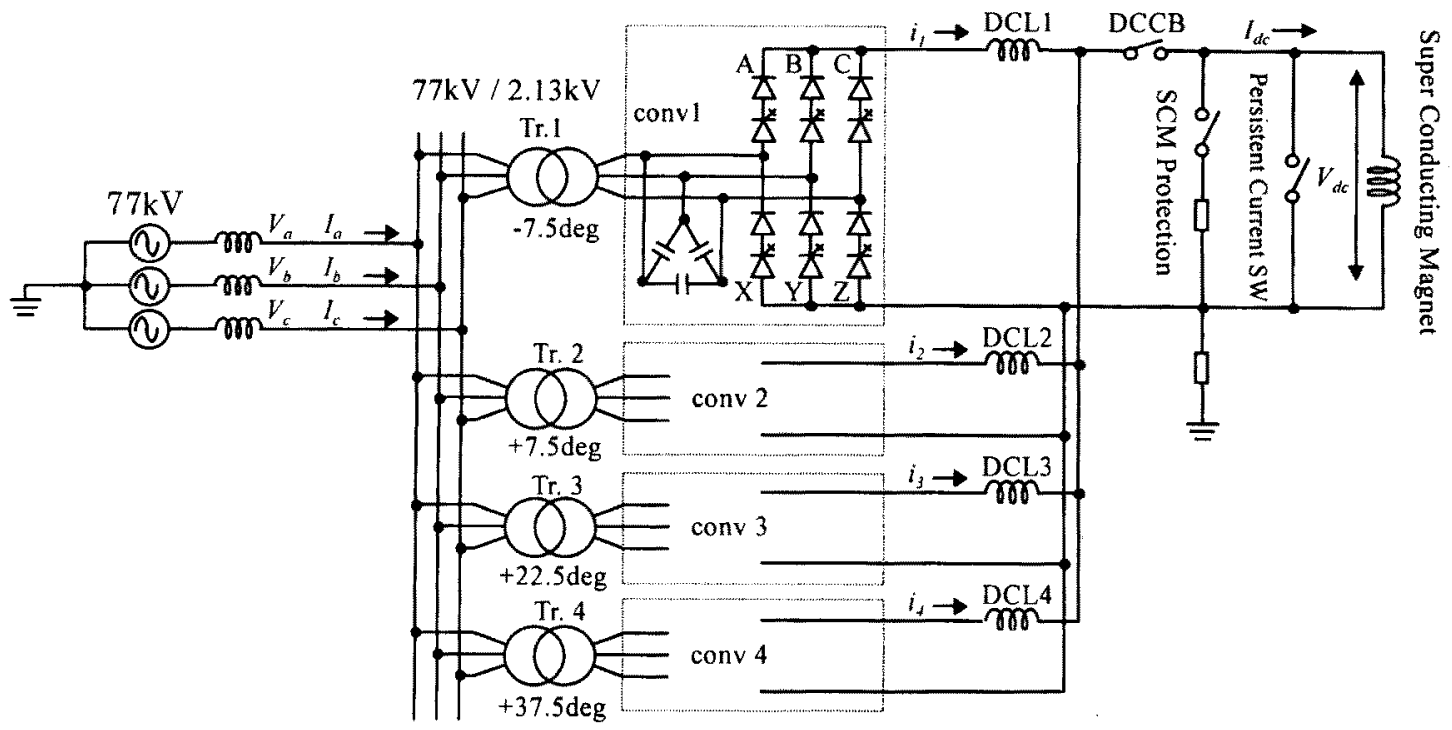

Fig. 1. Main circuit configuration of multiple current source converters 


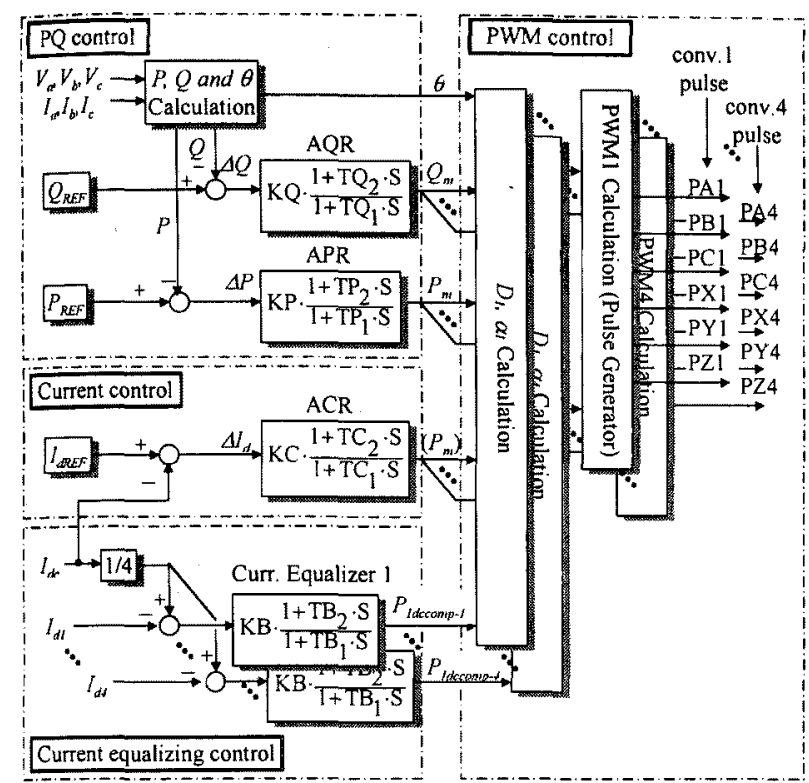

Fig. 2. Control block diagram

connected in parallel and $6 \mathrm{kV}-3 \mathrm{kA}$ blocking diodes connected in series to each GTO. Each transformer has difference phase, 15 deg., to eliminate the harmonic distortion. All transformers are connected in parallel to $77 \mathrm{kV}$ transmission line. AC capacitors are attached between the transformer and the converter to absorb transient overvoltage caused by the current commutation of the converter. The DC side of the converters is connected to the superconducting magnet (SCM) through DC reactors (DCLs') and DC circuit breaker (DCCB). The parallel connection of DCLs' reduces the ripples of the DC voltage and prevents the circulating current among the converters. There is a mechanical switch called a persistent current switch which makes a current path when DCCB is open that means the converters are not in the power conditioning mode of the transmission line. There is a discharging resistor connected across SCM for the protection reason when the quench occurs, which is breakdown of superconductivity.

\section{Converter Control System}

The converters store the electric energy of the transmission line into the SCM as the magnetic energy and discharge the magnetic energy into the transmission line as the electric energy, which is the real power $(P)$ control. Further the converters should stabilize the voltage of the transmission line, which is the reactive power $(Q)$ control. The controller of the converters should control $P$ and $Q$ simultaneously. Fig. 2 is a proposed schematic block diagram that illustrates how the controller works. There are several control blocks; Automatic Real Power Regulator (APR) and Automatic Reactive Power Regulator (AQR), Automatic Current Regulator (ACR), Current equalizer that controls the current balance among the converters and the pulse generator that gives PWM pulse to the converters. These control blocks fill the function as follows.

\section{(i) $P Q$ Control and the voltage phase angle $\theta$ calculation}

$P$ and $Q$ out of the converters are detected by equation (1)-(3). After $P$ and $Q$ are compared with the references $P_{r e f}$ and $Q_{r e f}, P_{m}$ and $Q_{m}$ are given through APR and $\mathrm{AQR}$.

The transformation from abc phase variables to $\alpha \beta$ variables can be written in the following matrix form:

$$
\begin{aligned}
& \left(\begin{array}{l}
V_{\alpha} \\
V_{\beta}
\end{array}\right)=\left(\begin{array}{ccc}
1 & -\frac{1}{2} & -\frac{1}{2} \\
0 & \frac{\sqrt{3}}{2} & -\frac{\sqrt{3}}{2}
\end{array}\right) \cdot\left(\begin{array}{l}
V_{a} \\
V_{b} \\
V_{c}
\end{array}\right) \\
& \left(\begin{array}{l}
I_{\alpha} \\
I_{\beta}
\end{array}\right)=\left(\begin{array}{ccc}
1 & -\frac{1}{2} & -\frac{1}{2} \\
0 & \frac{\sqrt{3}}{2} & -\frac{\sqrt{3}}{2}
\end{array}\right) \cdot\left(\begin{array}{l}
I_{a} \\
I_{b} \\
I_{c}
\end{array}\right)
\end{aligned}
$$

Then $P$ and $Q$ are given by,

$$
\left(\begin{array}{l}
P \\
Q
\end{array}\right)=\left(\begin{array}{cc}
V_{\alpha} & V_{\beta} \\
-V_{\beta} & V_{\alpha}
\end{array}\right) \cdot\left(\begin{array}{l}
I_{\alpha} \\
I_{\beta}
\end{array}\right)
$$

The voltage phase angle $\theta^{[2]}$ of $V_{a}$ of the transmission line is also calculated. The angle speed detection is very important, because every calculation in the control block is based on it. The control quantity after $\mathrm{APR}$ and $\mathrm{AQR}$ is transferred to PWM block to make PWM switching pulse. $\theta$ of $V_{a}$ is given by following equations (4) (6).

$$
\begin{aligned}
& \left(\begin{array}{l}
I_{p} \\
I_{q}
\end{array}\right)=\left(\begin{array}{l}
P / V \\
Q / V
\end{array}\right) \\
& V=\sqrt{V_{\alpha}^{2}+V_{\beta}^{2}} \\
& \theta=\cos ^{-1}\left(\frac{\left|I_{p} \cdot I_{\beta}+I_{q} \cdot I_{\alpha}\right|}{I_{\alpha}^{2}+I_{\beta}^{2}}\right) \quad\left(I_{p} \cdot I_{\beta}+I_{q} \cdot I_{\alpha} \geq 0\right) \\
& \theta=-\cos ^{-1}\left(\frac{\left|I_{p} \cdot I_{\beta}+I_{q} \cdot I_{a}\right|}{I_{\alpha}^{2}+I_{\beta}^{2}}\right)\left(I_{p} \cdot I_{\beta}+I_{q} \cdot I_{\alpha}<0\right)
\end{aligned}
$$

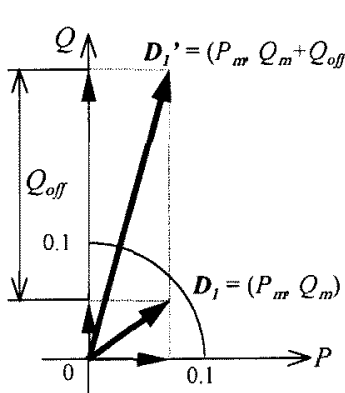

(a) converter 1 and 3

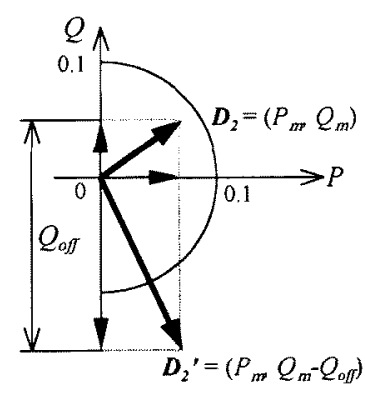

(b) converter 2 and 4

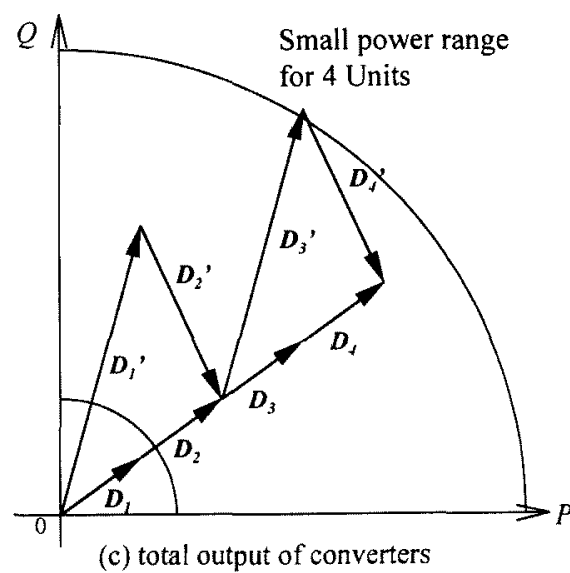

Fig. 3. Principle of small power output 
where $I_{p}$ and $I_{q}$ are real part and reactive part of the AC current respectably.

\section{(ii) A Control Method of Small Power Output}

There is an uncontrollable region in the center of the fourquadrant, because the minimum permissible switching pulse width should be larger than $100 \mu \mathrm{s}$ for $6 \mathrm{kV}-3 \mathrm{kA}$ GTO for the protection reason. A unique control method of the small power output is used to cover all the range of the four-quadrant which SMES system should control. Although a conventional single converter cannot cover this region, the proposed converters can control it with the advantage of the multiple structure. The proposed control theory is shown in fig. 3 .

In the normal power region, the control quantity of $P_{m}$ and $Q_{m}$ are distributed to PWM controllers equally as shown in equation (7).

$\boldsymbol{D}_{\boldsymbol{l}}=\boldsymbol{D}_{2}=\boldsymbol{D}_{3}=\boldsymbol{D}_{4}=\left(P_{m}, Q_{m}\right)$

where $\boldsymbol{D}_{n}(n: 1, \ldots, 4)$ are power amplitude which is expressed vectors of a direct and a quadrature factors and of which absolute amplitude is given as a next equation.

$\left|D_{n}\right|=\sqrt{P_{m}^{2}+Q_{m}^{2}}$

In the small power region $\left(\mid D_{n} \leq 0.1\right)$, the originally calculated $P_{m}$ and $Q_{m}$ are shifted; $Q_{m}$ changed into $Q_{m}+Q_{o f f}$ for converter 1 and 3 , and $Q_{m}$ changed into $Q_{m}-Q_{o f f}$ for converter 2 and 4 respectively as shown in equations (9) and (10). This procedure is also shown in fig. 3(a) and (b). It is possible to shift each vector of converter output by adding or subtracting reactive power without changing the DC current of each converter. $Q_{o f f}$ is required larger than 0.2 in this system. Even though total $D$ is smaller than $0.1, D_{n}$ are always larger than 0.1 as shown in fig. 3(c). PWM controllers, therefore, never output the pulses less than $100 \mu \mathrm{s}$.

$\boldsymbol{D}_{l}=\boldsymbol{D}_{3}=\left(P_{m}, Q_{m}\right) \rightarrow \boldsymbol{D}_{l}^{\prime}=\boldsymbol{D}_{3}^{\prime}=\left(P_{m}, Q_{m}+Q_{o f f}\right)$

$\boldsymbol{D}_{2}=\boldsymbol{D}_{1}=\left(P_{m}, Q_{m}\right) \rightarrow \boldsymbol{D}_{2}^{\prime}=\boldsymbol{D}_{1}^{\prime}=\left(P_{m}, Q_{m}-Q_{n f f}\right)$

where $Q_{o f f} \geq 0.2$

\section{(iii) Current Equalizing Control}

For the current source multiple structured converters, the imbalance of the DC current among the converters will happen due to the dispersion of various parameters such as the impedance in the converters and the leakage reactance of the transformers. This problem will not only deteriorate utilization rate and the lifetime of each element but also will make the control system unstable. The current equalizing control is necessary as a minor loop of APR and $\mathrm{AQR}$. A quarter of the SCM current is given as the reference signal to the Current Equalizer as shown in fig. 2. Those signals are calculated through PI controller for each converter. The control quantity from the Current Equalizer is dimension of real power at this stage. The control quantity for the real power is replaced with the next equation.

$$
P_{m-n} \rightarrow P_{m}+P_{\text {idcomp.n }}(n: 1, \ldots, 4)
$$

where $P_{m-n}$ is control quantity of real power for each $D_{n}$ and $\alpha_{n}$ calculation block

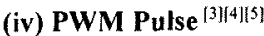

PWM control method is very important for a current source converter as well as a voltage source converter, because it decides the large part of the loss of the converters and the harmonic distortion included in the output current of the converters.

While these two factors are contrary to each other, the efficient is more focused on than the harmonics in the connection of the

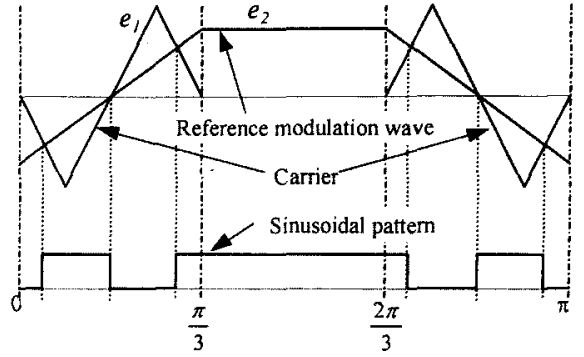

Fig. 4. PWM controlled current pattern

power transmission system, only because the power transmission system requires that total harmonic distortion is less than $5 \%$ and each harmonic component is less than $3 \%$. That is why 5 -pulse PWM control method is used to meet the both requirements in this paper.

PWM pulse is derived from $P_{m}, Q_{m}, P_{\text {idccomp-n }}$, and $\theta$ by following next procedure. Equation (8) and (11) are changed into (12).

$$
\begin{aligned}
& \left|D_{n}\right|=\sqrt{\left(P_{m}+P_{i d c c o m p-n}\right)^{2}+Q_{m}{ }^{2}} \\
& \alpha_{n}=\cos ^{-1}\left(\frac{\left|Q_{m}\right|}{\left|D_{n}\right|}\right) \quad\left(Q_{m} \geq 0\right) \\
& \alpha_{n}=-\cos ^{-1}\left(\frac{\left|Q_{m}\right|}{\left|D_{n}\right|}\right) \quad\left(Q_{m}<0\right)
\end{aligned}
$$

where $Q_{m}$ is replaced with $Q_{m} \pm Q_{\text {off }}$ when small power output.

$\alpha$ is power factor and is used with $\theta$ to synchronized with the angle of the transmission line. These $D_{n}, \alpha$ and $\theta$ are applied in the

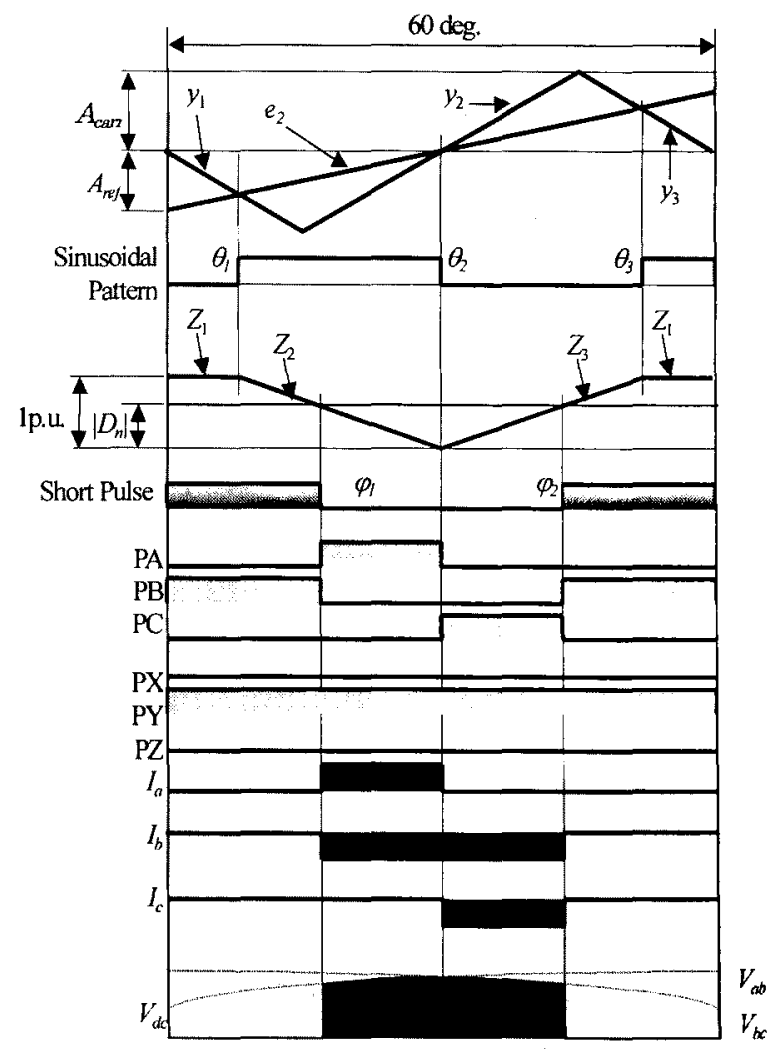

Fig. 5. Principle of PWM control method 
PWM controllers. It tries to make the AC output current sinusoidal by the comparison between a carrier $e_{I}$ and a reference wave $e_{2}$ as shown in fig. 4 which shows the first half of the electric cycle. As the electric cycle can be divided into six parts, this method is explained with the first period $(0 \leq \theta \leq \pi / 3)$ as an example of $\alpha=$ 0 . The reference wave as shown in fig. 5 is given by equation (14).

$$
e_{2}=A_{\text {ref }} \cdot\left(\frac{6}{\pi} \cdot \theta-1\right)
$$

where $A_{r e f}\left(0<A_{r e f} \leq 1\right)$ is the amplitude of the reference wave.

The carrier is expressed with three parts in this period, which are given in equations (15)

$$
\begin{array}{ll}
y_{1}=-\frac{12}{\pi} \cdot \theta & (0 \leq \theta<\pi / 12) \\
y_{2}=\frac{12}{\pi} \cdot\left(\theta-\frac{\pi}{6}\right) & (\pi / 12 \leq \theta<\pi / 4) \\
y_{3}=-\frac{12}{\pi} \cdot\left(\theta-\frac{\pi}{3}\right) & (\pi / 4 \leq \theta<\pi / 3)
\end{array}
$$

The cross points of these waveforms are given by following conditions,

$$
e_{2}=y_{1}, \quad e_{2}=y_{2}, \quad e_{2}=y_{3}
$$

Therefore,

$$
\theta_{1}=\frac{\pi}{6} \cdot \frac{A_{r e f}}{A_{r e f}+2}, \theta_{2}=\frac{\pi}{6}, \theta_{3}=\frac{\pi}{6} \cdot \frac{A_{r e f}+4}{A_{r e f}+2}
$$

Synchronizing the rise and the down edges of sinusoidal pattern that is referring to fig. 4 , the $\mathrm{V}$-shape wave $V_{W M}$ is made. $V_{W M}$ is expressed with three equations.

$$
\begin{array}{ll}
Z_{1}=1 & \left(0 \leq \theta \leq \theta_{1}, \theta 3 \leq \theta \leq \pi / 3\right) \\
Z_{2}=-\frac{3 \cdot\left(A_{r e f}+2\right)}{\pi} \cdot\left(\theta-\frac{\pi}{6}\right) & \left(\theta_{1} \leq \theta<\pi / 6\right) \\
Z_{3}=\frac{3 \cdot\left(A_{\text {ref }}+2\right)}{\pi} \cdot\left(\theta-\frac{\pi}{6}\right) & \left(\pi / 6 \leq \theta<\theta_{3}\right) \ldots \ldots(18)
\end{array}
$$

The short pulses are necessary to circulate the DC current at all time. The short pulses are made from the comparison between $V_{W M}$ and $D_{n}$. The cross points of $V_{w M}$ and $D$ are given as the functions of $D_{n}$ and $A_{\text {ref }}$

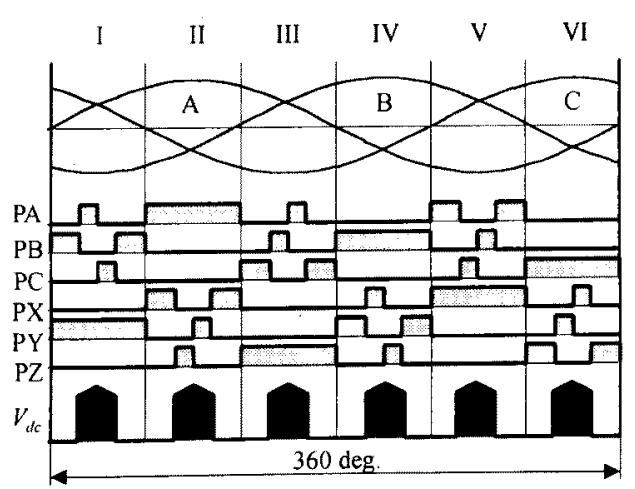

Fig. 6. PWM Pulse and $V_{d c}$

電学論 D, 120 巻 3 号, 平成 12 年

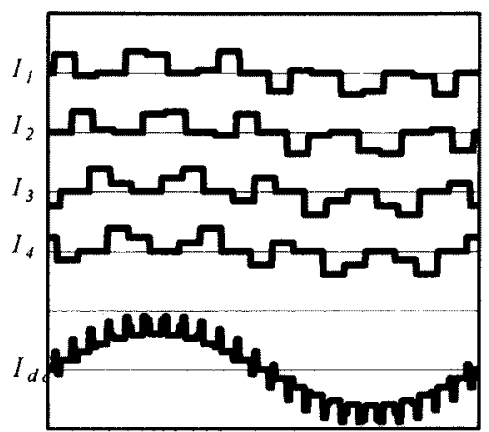

Fig. 7. Current waveforms $\left(A_{r e f}=0.7, D n=0.8\right)$

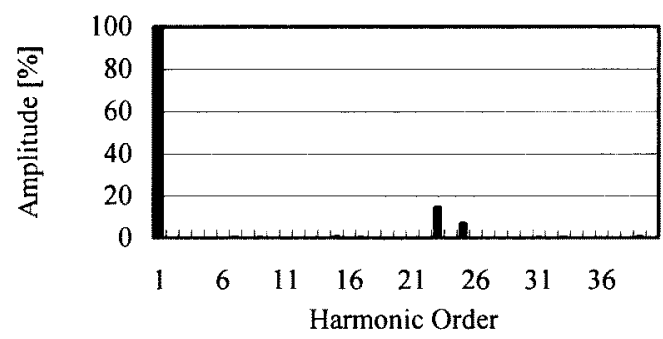

Fig. 8. Harmonics of total current waveform $\left(A_{r e f}=0.7\right.$,

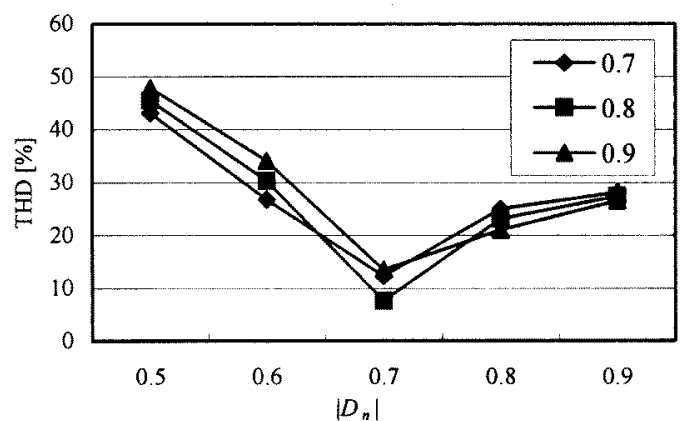

Fig.9. THD as function of $D_{n}$ with variation of $A_{\text {ref }}$

$$
\varphi_{1}=\frac{\pi}{6}-D_{n} \cdot \frac{\pi}{3 \cdot\left(A_{r e f}+2\right)}, \varphi_{2}=\frac{\pi}{6}+D_{n} \cdot \frac{\pi}{3 \cdot\left(A_{r e f}+2\right)}
$$

Further all switching pulses are derived from these two cross points and a following principle as shown in fig. 5. The short pulses are defined as

$$
\text { short pulse }=\left\{\begin{array}{l}
1 \cdots 0 \leq \theta<\varphi_{1}, \varphi_{2} \leq \theta \leq \pi / 3 \\
0 \cdots \varphi_{1} \leq \theta<\varphi_{2}
\end{array}\right.
$$

PB is the same as the short pulse. While PA follows the left half side of the opposite state to the short pulses,

$$
\mathrm{PA}=\left\{\begin{array}{l}
1 \cdots \varphi_{1}<\theta \leq \pi / 6 \\
0 \cdots 0 \leq \theta \leq \varphi_{1}, \pi / 6<\theta \leq \pi / 3
\end{array}\right.
$$

PC follows the right half side 


$$
\mathrm{PC}=\left\{\begin{array}{l}
1 \cdots \pi / 6<\theta \leq \varphi_{2} \\
0 \cdots 0 \leq \theta \leq \pi / 6, \varphi_{2}<\theta \leq \pi / 3
\end{array}\right.
$$

$\mathrm{PX}$ and $\mathrm{PZ}$ are off, and $\mathrm{PY}$ is on.

$$
\begin{aligned}
& \mathrm{PX}=1 \cdots 0 \leq \theta \leq \pi / 3 \\
& \mathrm{PY}=0 \cdots 0 \leq \theta \leq \pi / 3 \\
& \mathrm{PZ}=1 \cdots 0 \leq \theta \leq \pi / 3
\end{aligned}
$$

As these switching pulses rectify the $\mathrm{AC}$ input at the cross point of $V_{a b}-V_{b c}$, the converters give the DC voltage to SCM. DC voltage and $\mathrm{AC}$ currents should be zero during the short pulses. DC voltage ripples are reduced by the connection of DCLs. AC current harmonics are absorbed by AC capacitors. This procedure will cover II-VI periods with different angle respectively as shown in fig. 6 .

Fig. 7 shows the typical waveforms of each converters' current and total output current at $77 \mathrm{kV}$ line of the transformers at the condition of $A_{r e f}=0.7, D_{n}=0.8$. Although the difference from the waveforms by the principle is due to the windings of the transformers which shift the angle $-7.5,+7.5,+22.5$ and +37.5 degree respectively as shown in equations (26), the angle of base frequency wave in each waveform matches perfectly.

Conv. 1: $\theta \rightarrow \theta+7.5^{\circ}$

Conv. 2: $\theta \rightarrow \theta-7.5^{\circ}$

Conv. 3: $\theta \rightarrow \theta-22.5^{\circ}$

Conv. $4: \theta \rightarrow \theta-37.5^{\circ}$

Fig. 8 shows the spectrum of total current wave at $A_{\text {ref }}=0.7$ and $D_{n}$ $=0.8$, that shows lower harmonic distortion is eliminated by transformer winding. THD as a function of $D_{n}$ with variation of $A_{\text {ref }}$ $(0.7,0.8$ and 0.9$)$ is shown in Fig.9. Although $D_{n}=0.7$ at $A_{r e f}=0.8$ gives the smallest value, $A_{r e f}=0.7$ is used in this system because THD of $A_{r e f}=0.7$ gives smallest figure for the wide range of $D_{n}$ $=0.5$ to 0.9

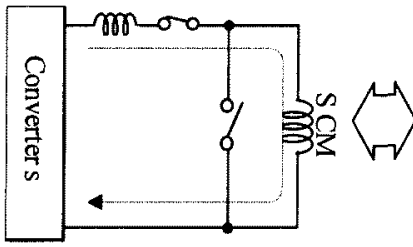

(1) Power Control Mode

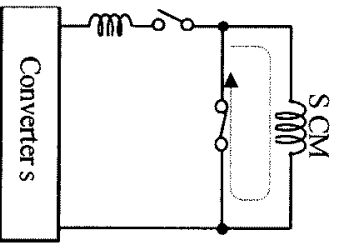

(2) Stand-by Mode
Fig. 10. Main Circuit Operation Mode

\section{(v) Current Control}

In the practical operation, the converters are not always conditioning the transmission line, for example, at the time of the initial current preservation of SCM. As seen in the control block diagram, SCM current is compared with the current reference and treated in an automatic current regulator (ACR) with PI controller. The current error correction signal is given as $P_{m}$ signal, because it charges and discharges real power. $Q_{m}$ is given as zero to avoid any influence of reactive power on the transmission line. PWM controllers give the switching pulse by the same procedure as power conditioning mode.

This control method is also effective in cooperating DCCB and the persistent current switch operation. When the converters do not control the power condition, SCM is not necessary to be connected to converters to avoid the loss by the switching. To keep SMES energy inside, a current loop that the SCM current flows through is
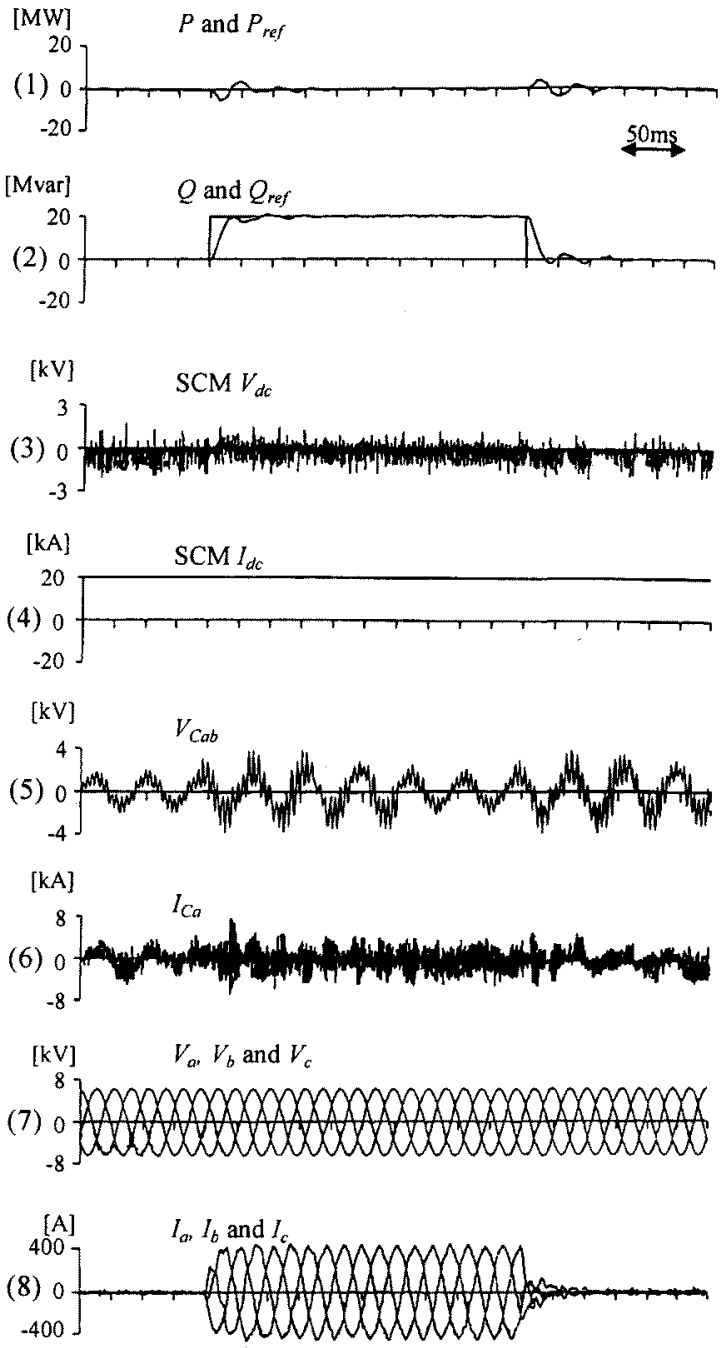

(1) $P$ and $P$ reference, (2) $Q$ and $Q$ reference, (3) SCM voltage, (4) SCM current, (5) AC voltage across AC capacitor, (6) AC capacitor current (7) AC voltage of transmission line, and (8) $\mathrm{AC}$ current of transmission line

Fig. 11. Waveforms of steady state Q step response

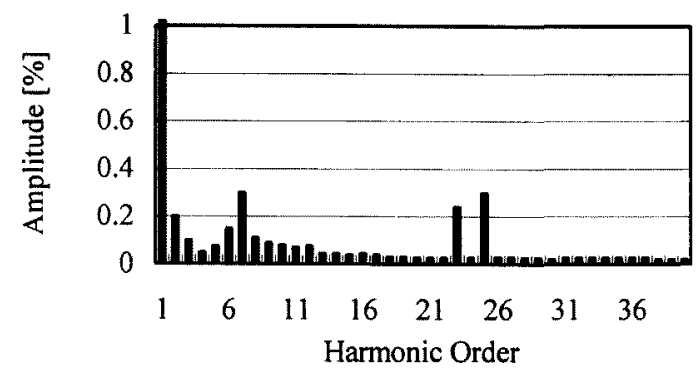

Fig. 12. Harmonics of $77 \mathrm{kV}$ voltage

necessary with extremely small resistance. Fig. 10 shows how the circuits are changed. First the converters are shifted to a short circuit state, then persistent current switch turns on. It is a little voltage across persistent current switch, because most of the current is flowing in the converters initially. The switch current is increased gradually by controlling DC current of the converters. Considering the lifetime of the switch, this cooperation is important. 
4. Simulation Results

Simulations are taken place under the condition of tablel.

Table 1. Simulation parameters

(i) Transmission line

\begin{tabular}{l|l}
\hline Capacity of $77 \mathrm{kV}$ transmission line & $600 \mathrm{MVA}$ \\
\hline (ii) Converters & $20 \mathrm{MVA}$ \\
\hline Capacity & $2.13 \mathrm{kV}$ \\
\hline AC voltage & $600 \mu \mathrm{F} /$ phase \\
\hline $\mathrm{AC}$ capacitor & $2 \mathrm{mH} /$ converter \\
\hline DCL & $1 \mathrm{kV}$ \\
\hline DC voltage & $20 \mathrm{kA}$ \\
\hline $\mathrm{DC}$ current & $4 \mathrm{H}$ \\
\hline SCM & \\
\hline (iii) Transformer & $20 \mathrm{MVA}$ \\
\hline Capacity & $10 \%$ on 20MVA base \\
\hline$\%$ impedance
\end{tabular}

Fig. 11 indicates a $Q$ step response. It is surveyed that the transmission line $\mathrm{Q}$ converges in the reference within $10 \mathrm{~ms}$. The interference to $\mathrm{P}$ is less than $10 \%$ as shown in fig. 11 (1). DC current is almost constant. AC capacitor and transformer impedance reduces the transient overvoltage across GTO to about $4 \mathrm{kV}$ to a maximum as seen from fig. 11 (5) that is within the usage of GTO peak maximum voltage. Under this control, THD on the $77 \mathrm{kV}$ line voltage is less than $1 \%$ (fig. 12), for this time THD is $0.6 \%(2 \mathrm{nd}-100 \mathrm{th})$, and the largest value of the harmonics included in the waveform is less than $0.3 \%$.

The waveforms under the small power control are shown in fig. 13. The waveforms (1)-(4) are $P$ and $Q$ control quantity signals given to PWM pulse generators. It confirms the $Q$ compensation method with changing the references $(P, Q)=(0,20 \mathrm{MVA})$ to $(0$, 4MVA). It can be said that $\left|D_{n}\right|$ value is always out of 0.1 p.u. At this time $I_{d c}$ is almost constant and it can be found good convergence between the power reference and the transmission line power. Even the transition time of $Q$, there is no oscillation of each $Q$ control quantity signal and total $Q$. The small power control is successfully operated.

When the converters are started without the current equalizing control and with adding impedance of $20 \mathrm{~m} \Omega$ in the converter 3 and 4 initially, the currents of converter 1 and converter 3 are diverged to $5500 \mathrm{~A}$ and $4500 \mathrm{~A}$ at about $100 \mathrm{~ms}$. The currents converge in same value of $5000 \mathrm{~A}$ within $400 \mathrm{~ms}$ after operating the equalizing control. There can be no effect on DC current and PQ value as seen from fig. $14(3)-(5)$. This shows this control method operates successfully in this system.

The controlled waveforms of persistent current switch closing and opening transition mode are shown in fig. 15. The persistent current switch is closed after the start of the current control mode, then the current of the converters are headed for zero with the guidance of the ramp reference. All current passes through persistent current switch. That completes the closing procedure. The waveforms also show the opening control after $350 \mathrm{~ms}$. The current of the converters is increased with ramp current reference. After the completion of the preservation of the current, the control mode is transit to $\mathrm{PQ}$ control mode $((\mathrm{P}, \mathrm{Q})=(20 \mathrm{MW}, 0))$. $\mathrm{P}$ is increased gradually to $20 \mathrm{MW}$. It can be found good accordance with that reference.

\section{Conclusion}

Multiple structured current-source converters are proposed for the SMES system. The control methods for the practical SMES

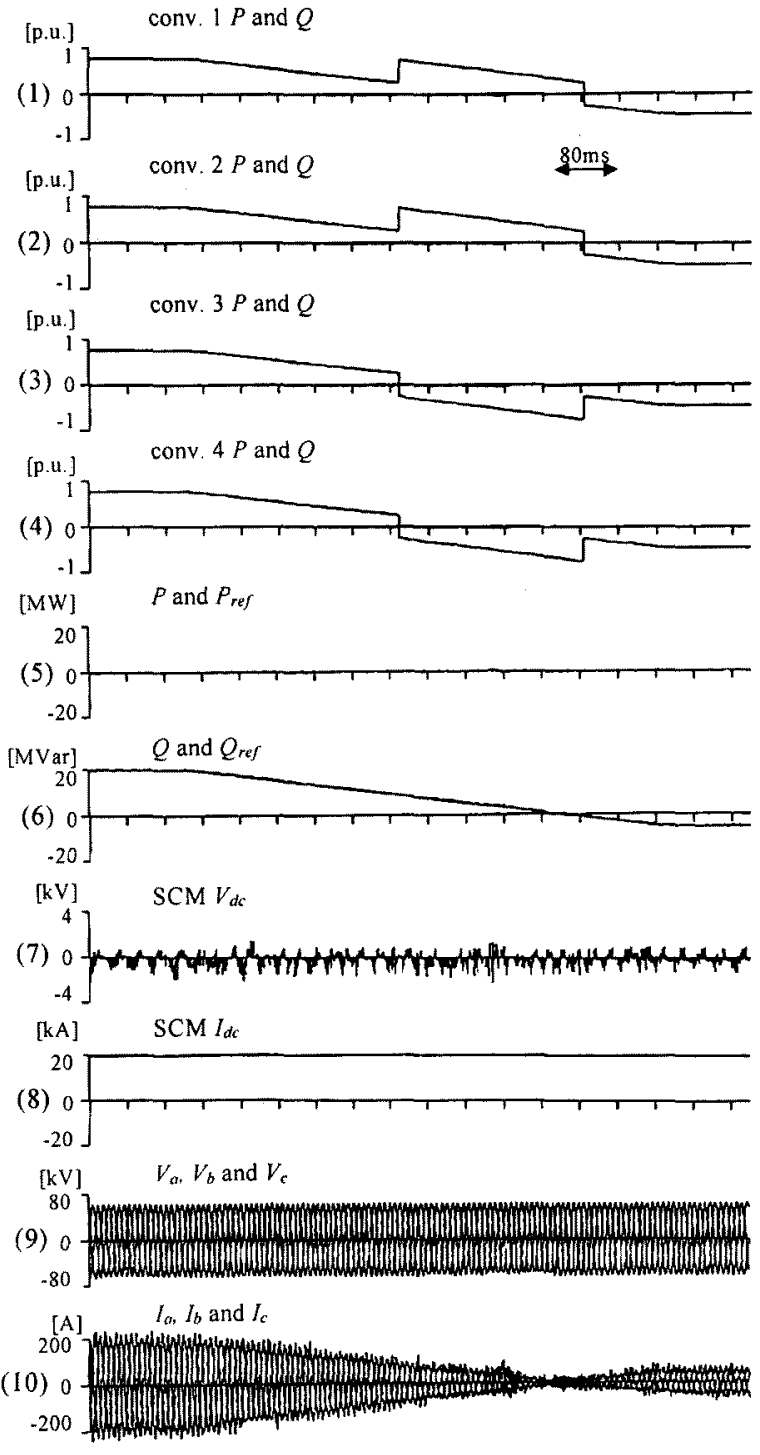

(1) $P$ and $Q$ reference of conv. 1, (2) $P$ and $Q$ reference of conv. 2, (3) $P$ and $Q$ reference of conv. 3, (4) $P$ and $Q$ reference of conv. 4, (5) $P$ and $P$ reference, (6) $Q$ and $Q$ reference (7) SCM voltage, (8) SCM current, (9) AC voltage of transmission line, and (10) AC current of transmission line

Fig. 13. Waveforms of small power output

system application are explained and the simulation results assuming the actual operation indicate their feasibility. Control method and controller achieved stable output for transmission line. It is found SMES system operation with the rapid response of AQR and $\mathrm{APR}$, and the low distortion output voltage for the power conditioning in the steady state. The digital simulation results have been presented and evaluated of which the operations work successfully. Then it can be confirmed that the realization of the proposed 20MW SMES system pilot plant. 


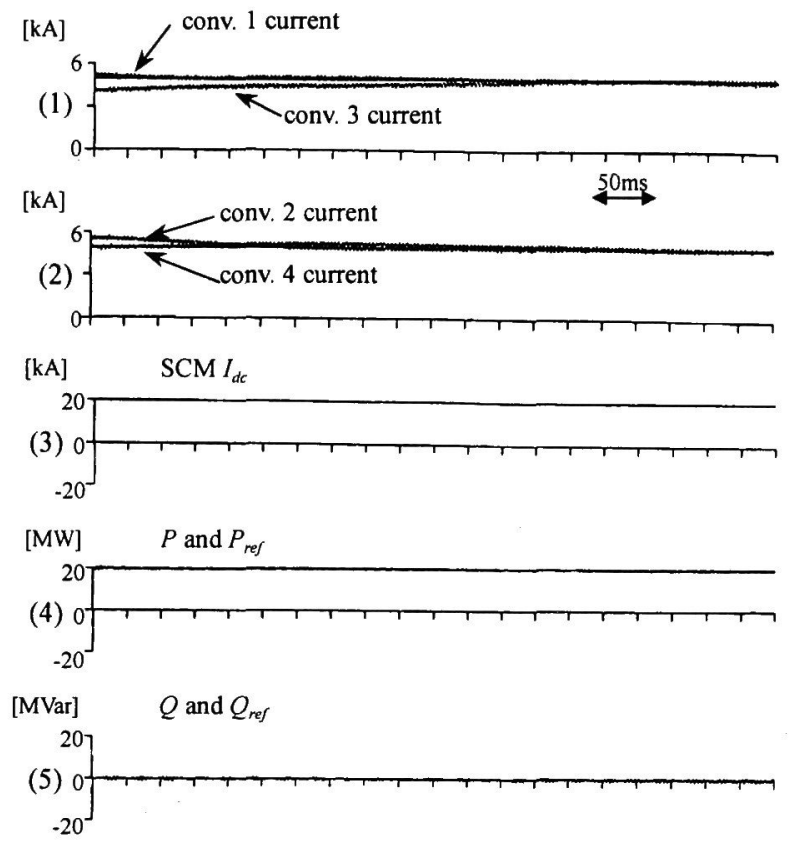

(1) DC current of conv. 1 and 3, (2) DC current of conv. 2 and 4, (3) SCM current, (4) $P$ and $P$ reference, and (5) $Q$ and $Q$ reference

Fig. 14. Waveforms of DC current equalizing control
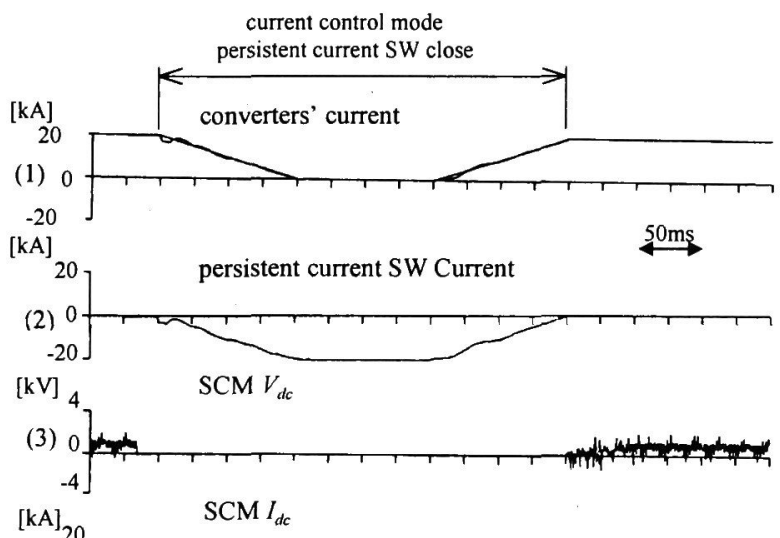

(4) 0

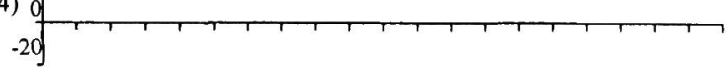

$[\mathrm{kV}]_{8} \quad V_{a}, V_{b}$ and $V_{c}$

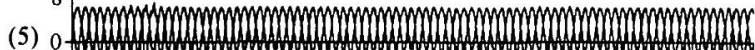

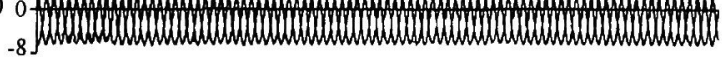

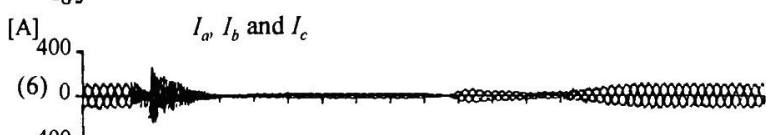
$-400$

(1) DC current of converters (2) DC current of persistent current SW, (3) SCM voltage, (4) SCM current, (5) AC voltage of transmission line, and (8) AC current of transmission line

Fig. 15. Waveforms of DC current control

(Manuscript received Jun, 7, '99, revised Sep., 21, '99)

\section{References}

[1] K. Imaie, et al, "Multiple Parallel Current-source PWM Converters for a 20MW SMES System", Japan Industry Applications Society Conference 1994, PE-10, pp85-90.

[2] Osami Tsukamoto, Yoshiaki Nagai and Kazuhiro Imaie "Direct Connected Multiple Current-source Converters for SMES system", IEE-Japan, Category D, Sept. 1998, pp992999.

[3] H. Konishi, et al, "A control method for self commutated power conversion system", IEE-Japan IAS National Meeting 1993, No.1273, pp9-297-9-298.

[4] Shirahama, et al, "Instantaneous Control Method with a GTO Converter for Active and Reactive Powers in Superconducting Magnetic Storage", PESC '88 record April 1988, I d-1, pp.101107.

[5] A. Ueda, et al, " Control Method and Characteristics of Current Source GTO Converter with Sinusoidal Input Current ", IEE-Japan, Category D, Nov. 1987, vol07-D, No.11, pp 1316-1323

[6] M. Honbu, et al, "PWM Control Method on Current Source GTO Inverter with Sinusoidal Output", IEE-Japan, Category D, Jul. 1986, vol06, No.7, pp25-32

Kazuhiro Imaie (Member) received his MSEE degree from

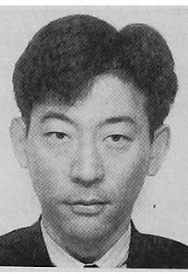
Doshisha University in 1990 . He has been worked as a Systems engineer in Power Electronics Design and Manufacturing Department in Hitachi Ltd. after graduation. He finished Power System Engineering Course in General Electric Co. in 1996. He is presently responsible for the development of PCS for Battery Energy Storage Systems. He is a member of the IEEE.

Osami Tsukamoto (Member) received a Doctor of Engineering from the University of Tokyo in 1970 . He became a Lecturer 1970-1971, an Associate Professor 1971 - 1983 and is a Professor from 1984 at faculty of Engineering, Yokohama National University. His main research interests are Applied Superconductivity and Electrical Machine Control. He is a member of the Japan Association of Cryogenic Engineering.

Sakae Washida (non-member) received his BS and MS degrees in electrical engineering from Hokkaido University in 1983 and 1985 respectively. $\mathrm{He}$ joined Electric Power Development Co., LTD (EPDC) in 1985 . He is presently responsible for the development of SMES in New Energy \& Technology Development Department. 